\title{
Metal Oxide Sensor for Petroleum Industry Applications
}

\author{
Ahsan-Ul-Haq Qurashi \\ King Fahd University of Petroleum\& Minerals Center of Excellence in Nanotechnology \\ Chemistry Department \\ Dhahran, 31261Saudi Arabia \\ e-mail: ahsanulhaq@kfupm.edu.sa
}

\section{Introduction}

The primary stimulus on semiconductor gas sensors was given by Heiland [1], Bielanski et al. [2], and Seiyama et al. [3] and the important step was taken when Taguchi developed semiconductor sensors based on metal oxides as an industrial product (Taguchi-type sensors) [4]. Metal oxide based gas sensors attained tremendous interests since the first discovery in 1962 by Seiyama et al. [4] and Taguchi [5]. In semiconductor based gas sensors, atoms and molecules interacting with semiconductor surfaces influence surface properties of semiconductors, such as conductivity and surface potential [6-12]. Depending upon the nature of gases (oxidising or reducing) and type of materials ( $\mathrm{p}$-type or n-type semiconductor) electrical resistance either decrease or increase and recorded accordingly.

One of the most significant components of sensor is the sensing material. Thus the choice of appropriate materials is extremely important for the development of good sensors in terms of sensitivity, selectivity, reversibility, sustainability, dynamic range, and etc. Nanostructured materials present paramount advantages to facilitate integration and miniaturization of the devices. Controlling the size, shape, and structure of inorganic nanomaterials to search for new properties has become one of the major objectives of nanoscale science and technology, because of their structure, size, and shapedependent characteristics and novel electronic, magnetic, optical, chemical, catalytic, and mechanical properties that cannot be obtained in their bulk counterparts. Crystalline mesoporous nanostructured metal oxides with narrow pore size distributions and controllable morphologies have attracted huge interests in a wide range of applications such as gas sensing, optics or catalysis. Various interesting techniques have been used for the fabrication of mesoporous metal oxide nanostructures which include nanocasting, anodization, templating, metal templating, etc

The high surface area and thermal stability of the metal oxide materials instantly created interests in using these materials for efficient sensors. More recently, mesoporous and mesostructured materials have been recognized as promising candidates for the next-generation of optoelectronic miniatured devices. These characteristic features of metal oxide nanostructures potentially make them ideal candidates for efficient gas sensors.

\section{Metal oxide nanostructures}

A complex synthesis of $\operatorname{In}_{2} \mathrm{O}_{3}\left(\mathrm{SnO}_{2}\right.$, and $\left.\mathrm{TiO}_{2}\right)$ nanostructures with well-defined shapes are of special interest to understand basic sizedependent scaling laws and may be useful in a wide range of application fields, including photonics, nanoelectronics, information storage gas sensors, chemical and biosensors and catalysis. In this work we report controlled growth of $\ln _{2} \mathrm{O}_{3}$ nanostructures by simple chemical vapor deposition and thermal evaporation methods. The fabrication of largescale $\quad \mathrm{In}_{2} \mathrm{O}_{3} \quad$ nanowires, nanopencils, nanoneedles, nanocolumns, nanosyringes, hierarchical structures, nanopushpins, nanopyramids, octahedrons, nanorods, nanotrees, patterned nanowires etc. were realized. These larger families of $\ln _{2} \mathrm{O}_{3}$ nanostructures were attained at very low temperature $\left(\sim 250-800^{\circ} \mathrm{C}\right)$. Structural analysis reveals that these $\ln _{2} \mathrm{O}_{3}$ nanostructures are body centered cubic (bcc), with single crystal structure. TEM analysis showed that all different types of $\ln _{2} \mathrm{O}_{3}$ nanostructures are single crystalline in nature. Raman and PL spectrum also showed that these wide varieties of $\ln _{2} \mathrm{O}_{3}$ 
nanostructures have promising optical properties. It is noteworthy to mention that we are the first to develop large varieties of $\mathrm{In}_{2} \mathrm{O}_{3}$ nanostructures in grams quantity by reducing the overall growth temperature up to $250^{\circ} \mathrm{C}$ by a novel modified thermal evaporation and vapor transport techniques. For the first time horizontal and vertical nanopatterning of 1D $\quad \mathrm{In}_{2} \mathrm{O}_{3}$ nanostructures is accomplished. On the basis of experimental parameters a possible growth mechanism for the formation of $\ln _{2} \mathrm{O}_{3}$ nanostructures was proposed. The complex $\mathrm{In}_{2} \mathrm{O}_{3}$ nanostructures with high crystal quality provided new building blocks in future architecture functional nanodevices. These $\mathrm{In}_{2} \mathrm{O}_{3}$ nanostructures also have potential applications in ultrasensitive gas sensors, chemical and biosensor devices, where well defined easily accessible crystal surface is required. Hydrogen sensor response was measured for different types of $\ln _{2} \mathrm{O}_{3}$ nanostructures and showed promising response and recovery time. Figure 1 shows FESEM image of indium oxide nanostructures grown for different temperatures. Figure 2 shows XRD spectrum of In2O3 nanowires. Furthermore the fabrication of sensor devices based on $\ln _{2} \mathrm{O}_{3}$ nanostructures is under the investigation.

\section{Petroleum industry}

Metal oxides are promising materials for gas sensors for petroleum industry due to several reasons. These oxide materials are highly stable and can respond in harsh atmosphere. Air quality monitoring is difficult in harsh environmental conditions. Petroleum industry is keenly looking for highly stable sensing materials with long term stability to monitor ambient air, composition of effluents and the gases coming from the combustion. The chemical moieties are mainly $\mathrm{SO}_{2}, \mathrm{H}_{2} \mathrm{~S}, \mathrm{CO}_{2}$,
$\mathrm{CO}$ and VOC. Especially detection of $\mathrm{H}_{2} \mathrm{~S}$ and $\mathrm{SO}_{2}$ based sensors are paramount to petroleum industry.

The current material and technology in use put limitation in term of maintenance, reliability and sensitivity. Petroleum industry is looking more sensitive options in terms of detecting low quantity (ppm) and reliability (accuracy). The current options being used by petroleum industry some time fails to differentiate between some materials of close chemistry for instance $\mathrm{H}_{2} \mathrm{~S}$ and other sulphur gases at ppm level.

\section{References}

[1] G. Heiland, Zum Einfluss von Zeit. Phys. 138 (1954) 459-464.

[2] A. Bielanski, J. Deren, J. Haber, Nature 179 (1957) 668-669.

[3] T. Seiyama, A. Kato, K. Fujiishi, M. Nagatani, Anal. Chem. 34 (1962), 15021503.

[4] N. Taguchi, Published patent application in Japan, S37-47677, October (1962).

[5] W. Göpel and K. D. Schierbaum, Sensors and Actuators B, 26, (1995), 1-12.

[6] D. E.Williams, Solid State Gas Sensors, Adam Hilger, Bristol, UK, (1987) 71-123.

[7] K. Ihokura and J. Watson, CRC Press, Boca Raton, Fla, USA, (1994)

[8] S. R. Morrison, JohnWiley \& Sons, New York, NY, USA, 1994.

[9] H. V. Shurmer, J. W. Gardner, and H. T. Chan, Sensors and Actuators, 18, (1989) 361-371.

[10] J. W. Gardner and P. N. Bartlett, Measurement Science and Technology, 7, (2000) 1087.

[11] R.Menzel and J. Goschnick Sensors and Actuators B, 68, (2000) 115-122. 